\title{
Tradeoffs in Displaying Peripheral Information
}

\author{
Paul P. Maglio Christopher S. Campbell \\ IBM Almaden Research Center \\ 650 Harry Rd. \\ San Jose, CA 95120 USA \\ 408-927-2857 \\ \{pmaglio, ccampbel\}@almaden.ibm.com
}

\begin{abstract}
Peripheral information is information that is not central to a person's current task, but provides the person the opportunity to learn more, to do a better job, or to keep track of less important tasks. Though peripheral information displays are ubiquitous, they have been rarely studied. For computer users, a common peripheral display is a scrolling text display that provides announcements, sports scores, stock prices, or other news. In this paper, we investigate how to design peripheral displays so that they provide the most information while having the least impact on the user's performance on the main task. We report a series of experiments on scrolling displays aimed at examining tradeoffs between distraction of scrolling motion and memorability of information displayed. Overall, we found that continuously scrolling displays are more distracting than displays that start and stop, but information in both is remembered equally well. These results are summarized in a set of design recommendations.
\end{abstract}

\section{Keywords}

Peripheral information, dual-task tradeoffs, user interface design.

\section{INTRODUCTION}

With the widespread use of advertising banners on web pages designed to distract users and capture their attention, it is becoming increasingly important to understand the nature of interruptions and distraction in computer interfaces. Advertising is particularly insidious, as users have little control over what is displayed. In general, though, because computer users routinely leave open many applications while they work on one thing at a time, only a small amount of the information available on the computer screen is central to the user's current task [3]. But when mail arrives, when print jobs are finished, or when an application abnormally terminates, users often like to be informed. To take other examples, tips on how to use the

\footnotetext{
Permission to make digital or hard copies of all or part of this work for personal or classroom use is granted without fee provided that copies are not made or distributed for prolit or commercial advantage and that copies bear this notice and the full citation on the first page. To copy otherwise, to republish, to post on servers or to redistribute to lists, requires prior specific permission and/or a fee.

CHI '2000 The Hague, Amsterdam

Copyright ACM 2000 1-58113-216-6/00/04 $\ldots \$ 5.00$
}

current application displayed in a pop up window or news headlines displayed in a scrolling text display might be helpful, but are not strictly necessary. In configuring their systems to provide this sort of helpful information, users routinely balance their desire to be informed with their tolerance for being interrupted.

Some interfaces designed to assist users in automobile driving [1] or web navigation $[14,15,17]$ display recommendations through highlighting, annotation, sound, or speech. In these cases, the normal mechanism for driving or web browsing is still available, but the interfaces attempt to make the task easier and richer by providing additional information. Annotation in particular can be an effective means for conveying extra information about links on web pages without being distracting [2].

In designing a variety of user interfaces, the challenge is to create information displays that maximize information delivery while at the same time minimize intrusiveness or distraction. We call nonessential information peripheral information because it is not central to the current task, but might be helpful to it or informative in other ways. There are two ways in which information might be considered peripheral, in content and in display. Although peripheral information is ubiquitous, neither peripheral content nor peripheral displays have been systematically explored (but see [20]). In this paper, we are concerned mainly with issues of display.

In terms of content, the key to peripheral information is that it is not critical to task performance. Unlike what is generally studied in the literature on monitoring and supervisory control (e.g., [18]), inattention to peripheral information does not result in catastrophe, such as a nuclear meltdown or a plane crash. However, by providing peripheral information, a system offers a user the opportunity to learn more, to do a better job, or to keep track of less important tasks.

The term ambient information has been used to refer to subtle environmental cues when designed into systems to peripherally convey information such as network traffic (e.g., [9]). Specific environmental changes, such as the frequency of background noise or amount of background lighting, are associated with specific changes in system 
status. Of course, the design problem here is to make the mapping from system state to environmental state as obvious as possible and without interfering with the primary task [19].

Our goal in this paper is to consider what it takes to inform peripherally. Our approach is to view peripheral informing as imposing extra-task demands on a user's cognitive resources. In a computer context, this means a user attends to a primary task, such as reading an online document or editing text, but occasionally another item, such as a news story, becomes important and is briefly attended to. Specifically, the present study was performed to assess the mental workload tradeoffs and effectiveness of three singleline text displays (which we generally refer to as tickers) in conveying information under dual-task demands. Participants in our studies performed a primary text-editing task while at the same time monitoring headlines displayed on a ticker. We measured how distracting the tickers were on editing performance, and we measured how memorable the headlines were on a post-experiment test. In the end, we found that constantly scrolling tickers distract more than those that start and stop, but that some motion actually provides effective feedback, helping users efficiently schedule glances at the peripheral display. These results have implications for the design of a variety of user interfaces, including web pages, notification services, and help systems.

The paper is organized as follows. We first discuss scrolling ticker displays and some background theory. We next detail the three experiments run to test the tickers under dual-task conditions. We finally summarize the results of the experiments and draw conclusions or the design of systems that incorporate peripheral information.

\section{TICKER DISPLAYS}

The displays tested included a variety of tickers in three broad families: (a) continuous scrolling text (CS), (b) discrete scrolling text (DS), and (c) serial presentation (SP). In the CS case, text scrolls at a constant rate either from right to left (horizontal) or from top to bottom (vertical). In the DS case, text scrolls quickly to the center of display (either horizontally or vertically) where it stops for some period before scrolling off the display. In the SP case, the text does not scroll at all; rather, it is displayed in a constant position in the center of the display, each update replacing the last text with new text.

Despite the large number of possible tickers, only two classes have been systematically explored: horizontal CS and SP. Studies directly comparing horizontal CS and SP found no difference in comprehension for a display reading task [12]. Other studies comparing CS to static displays found that text is read more slowly [22] and is less comprehensible [8] when scrolling horizontally than when displayed on a static page. Further work showed that this effect does not depend on the number of words on the screen or on window size [5]. Studies comparing SP and static pages of text show that comprehension performance is about the same [10], and that reading latency is about the same as well [21]. Overall, these studies fail to show strong differences in comprehension among CS, SP, and static displays, and therefore cannot be used to make strong recommendations about display design.

A practical evaluation of scrolling displays as peripheral information displays requires an analysis of the mental workload involved in performing a primary task (such as text editing), and in shifting to a secondary task (reading the ticker). Since the tasks are performed concurrently, some type of scheduling strategy must be devised to determine the length of time to perform each task and the frequency of switching between tasks. Scheduling strategy or timesharing skills are one important determinant of performance in multi-task situations [23]. Evidence that scheduling strategy can influence multi-task performance independent of resource limitations comes from research showing improved dual-task performance after strategy training [13]. Additional evidence comes from studies showing improved performance after extended experience managing two tasks concurrently but with no improvements in performance on each task alone [4,7]. Task priority (relative payoff or relevance, see [10]) and bandwidth (frequency or pace of display) also influence scheduling strategy [24].

The present study concerns how visual display parameters can influence performance in a dual-task setting. Importantly, our participants were not told how to prioritize the two tasks or at what speed the display updates, either of which would help them create a strategy for scheduling their glances at the scrolling display. Many scheduling strategies are possible. One would be to watch the first few updates of the ticker display to establish a time span in which to edit then glance down at the ticker. Another strategy would be to edit until movement is detected in the periphery and then to look down to read the new headline, effectively using motion of the text displayed in the ticker as feedback to cue looking at the ticker.

Feedback the tickers provide, including motion and flashing, can help cue glances at the scrolling display. So we can order the tickers by the quality of feedback they provide users. Because the update of DS occurs gradually as the headline scrolls down to the center of the display, we believe that DS provides a better cue for shifting gaze to the ticker than SP, in which there is no motion. Because SP updates the display instantly and CS updates the display constantly, we believe that SP provides better feedback than CS. Thus, DS gives the best feedback, followed by SP, and then CS.

Along these lines, we hypothesized that (a) tickers with more motion would be more distracting, leading to fewer edits; and (b) tickers with less feedback would provide fewer cues for scheduling, leading either to fewer edits or to lower comprehension and retention of the ticker-displayed 
information.

The basic plan of our experiments is as follows. First, we tested the effects of three common tickers (horizontal continuous scrolling, vertical discrete scrolling, and serial presentation) on editing and memory performance to see whether the predicted ordering would obtain. Second, we switched the direction of motion for the scrolling displays to determine whether direction was relevant. Third, we tried alternative methods for providing update feedbackauditory and visual cues other than scrolling motion-to see whether feedback or motion dominates performance.

\section{EXPERIMENT 1}

The first experiment examined distraction versus memorability of three tickers. Distraction was defined as the change in performance for text editing alone versus text editing while concurrently reading the ticker display. The memorability of each display was defined as the number of displayed headlines recognized on a post-experiment multiple-choice test.

\section{Method \\ Participants first performed the text-editing task without the headline-reading task. Participants then performed both tasks. Performance on the editing task alone served as the baseline to compare to editing performance in the dual-task condition. After simultaneously performing the text-editing and headline-reading tasks, memorability was measured by a headline recognition test. More precisely, one between- subjects factor, ticker type, was manipulated across three levels: horizontal continuous scrolling (HCS), serial presentation (SP), and vertical discrete scrolling (VDS). Two dependent measures were collected for each participant: number of corrections made to the document and number of correct responses on the recognition test.}

\section{Participants}

Twenty-nine participants were recruited from IBM Almaden Research Center and compensated for their time.

\section{Materials}

The text editor and the ticker appeared as two separate displays on a computer screen (see Figure 1). The text edited by the participants was taken from a chapter of a scientific dissertation [16] and broken into two parts, which were counterbalanced in the two phases (i.e., with ticker/without ticker) of the experiment. As shown in Table 1 , both parts were of similar reading difficulty, as given by the Flesch ease of reading index and the Flesch-Kincad grade level [6].

Errors were introduced into the texts by hand according to three rules: (a) between 0 and 2 errors were put in each sentence; (b) errors were evenly spaced throughout the entire document; (c) errors included only subject-verb agreement, word order, and inconsistent verb tense. These error types were chosen (rather than spelling and other typographical errors) so that the editing task would be sufficiently demanding and thus produce a dual task

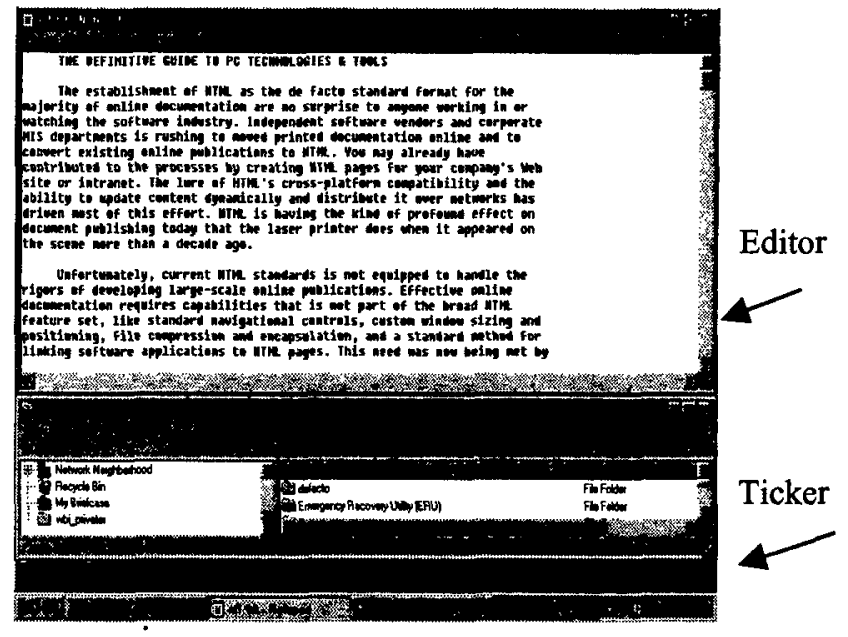

Figure 1: Screen shot showing editing window and ticker display for the dual-task conditions.

performance trade-off. Including typographical errors would make possible a text-skimming strategy. Skimming likely requires less effort than reading and is not the task we intended to test.

Table 1: Reading difficulty of edited documents.

\begin{tabular}{l|lc|cccc}
\hline & \multicolumn{4}{|c|}{ Experiment 1 } & \multicolumn{4}{c}{ Experiments 2 and 3 } \\
\hline Document & \multicolumn{1}{|c|}{$\mathbf{1}$} & $\mathbf{2}$ & $\mathbf{1}$ & $\mathbf{2}$ & $\mathbf{3}$ & $\mathbf{4}$ \\
\hline Total words & 1760 & 2027 & 2467 & 1580 & 2365 & 2060 \\
Words/sentence & 5.9 & 5.5 & 6.0 & 5.2 & 6.4 & 6.4 \\
$\begin{array}{l}\text { Reading ease } \\
\text { (Flesch) }\end{array}$ & 38.3 & 39.1 & 63.6 & 47.1 & 62.0 & 44.0 \\
$\begin{array}{l}\text { Grade level } \\
\text { (Flesch-Kincaid) }\end{array}$ & 9.3 & 9.1 & 5.8 & 7.9 & 6.2 & 8.7 \\
\hline
\end{tabular}

Reading ease ranges from 0 to 100 , with 0 being most difficult.

The tickers displayed thirty single-line headlines, averaging seven words apiece (see Table 2). The headlines were constructed as concise and self-contained summaries of news stories. The topics of the headlines were fictional but plausible. Each of the thirty headlines was displayed once in random order, and then this sequence repeated so that overall, each headline was displayed twice.

Table 2: Example headlines.

President Names Hoffman Secretary of Defense

French F-10 Fighter Jet Down Over Turkey

Finland Builds World's First Fusion Power Plant

The text for all ticker displays appeared in 17-point font with a cyan foreground and a black background. Each of 
these displays was 796 pixels wide by 24 pixels high. In the HCS condition, text continuously scrolled from right-toleft. The step size of each update was 5 pixels and the time between updates was $132 \mathrm{~ms}$. Each headline appeared in the information display window for 10 seconds. As soon as the last word of a headline had moved 10 pixels onto the display, the next headline began scrolling out. Headlines maintained a 10-pixel distance as they scrolled. For the SP condition, each headline was updated instantaneously and remained on the screen for 10 seconds before the next headline was presented. Each headline completely replaced the last one so that no two were on the screen at the same time. In the VDS condition, headlines scrolled from top-tobottom. Each headline scrolled down from the top for 333 $\mathrm{ms}$ and remained in the horizontal and vertical center of the screen for 9.666 seconds. When the next headline scrolled down from the top, the current headline scrolled from the center to the bottom and off the screen over a period of 333 ms.

\section{Procedure}

In the first phase, participants edited one of the two documents, making as many corrections as possible within ten minutes. They were told about the types of errors they could expect to find. After ten minutes, the second phase began, in which participants edited the other document and read the ticker display at the same time. The order in which the documents were presented was balanced across participants. Participants were told to make as many corrections as possible while reading the headlines. The importance of performing both tasks to the best of their ability was stressed, as well as the fact that they would be tested on their memory of the headlines. At the end of the ten-minute time limit, the experimenter administered the multiple-choice test. No time limit was enforced for completing this test.

\section{Results}

Editing performance was calculated as the percentage decrease in number of correct edits from the no ticker condition to the ticker condition for each participant. Based on this measure, three outliers-exceeding two standard deviations from the mean-were removed from consideration, leaving 10 participants in each of the HCS and SP groups, and 6 participants in the VDS group. The alpha level for this and all experiments was set at 0.05.

A paired samples t-test showed that number of corrections decreased significantly from the single- to the dual-task conditions, $t(25)=4.38, p<.001$. To test for differences in the effects of the three information displays, a one-way analysis of variance was calculated using percentage decrease in number of corrections as the dependent measure. A main effect for information display was marginally significant, $F(2,23)=3.20, p=0.059$. Figure 2 shows the mean percent decrease in the number of corrections made from the single-task to the dual-task condition. Planned comparisons using independent samples

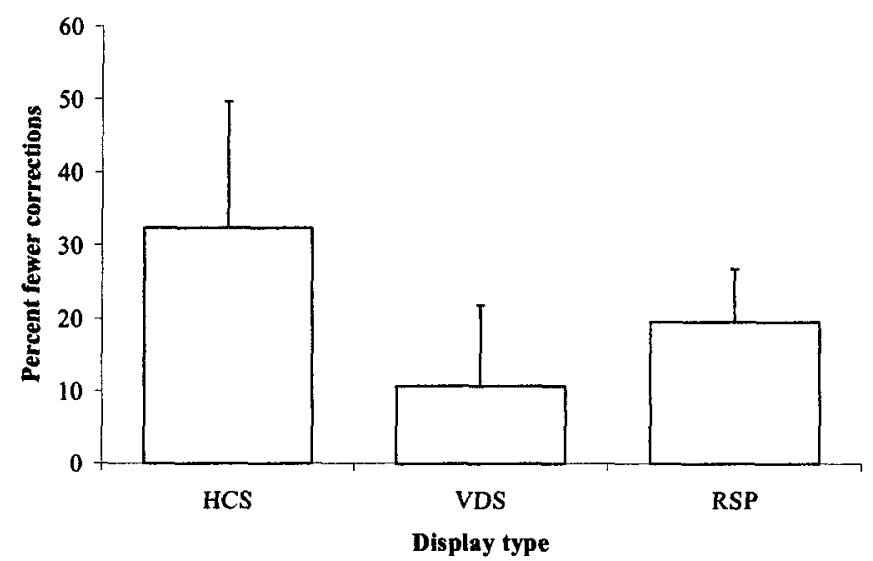

Figure 2: Mean percentage decrease in corrections between single- and dual-task conditions in Experiment 1.

$t$-tests (assuming unequal variance) indicated: (a) continuous scrolling (HCS) led to worse editing performance than discrete scrolling (VDS), $t(13)=2.46, p$ $=0.014$; (b) continuous scrolling (HCS) was marginally worse than serial presentation (SP), $t(12)=1.54, p=0.075$; and (c) serial presentation (SP) was marginally worse than discrete scrolling (VDS), $t(10)=1.64, p=0.066$.

To determine the memorability of the tickers, a one-way analysis of variance was calculated with percent correct on the multiple-choice test as the dependent variable. No difference was found among the displays, $F(2,23)=0.58$, NS. The failure to find significant differences was not the result of floor or ceiling effects, as scores spanned a normal range from $30 \%$ to $100 \%$ correct, with means of $70 \%$ for HCS, $67 \%$ for VDS, and $76 \%$ for SP.

\section{Discussion}

Though the differences in the number of corrections among information displays appear small during a 10-minute period, over the course of a full workday, productivity could be greatly influenced by the type of display chosen. For instance, using a continuous display rather than a discrete scrolling display, five fewer corrections were made every 10 minutes. If this rate of distraction is constant during four hours of editing, this would result in 100 fewer corrections for the continuous ticker, which could amount to several documents.

As mentioned, ordering the tickers by the quality of update feedback they provide, discrete scrolling gives the best feedback, followed by serial presentation, and then continuous scrolling. The ordering shown in Figure 2 is consistent with these predictions. More movement in the display (continuous scrolling) produces greater distraction and less feedback. At the same time, less movement (discrete scrolling) results in less distraction and more update feedback. These relationships suggest that distraction is a function of several factors, including amount of display movement and amount of feedback. 


\section{EXPERIMENT 2}

The results of Experiment 1 suggest that the discrete scrolling ticker provides the best balance of motion and update feedback, leading to least impact on concurrent task performance. However, because the discrete display scrolled vertically and the continuous display scrolled horizontally, we cannot rule out scroll direction as another factor affecting performance. The purpose of the second experiment was to rule out direction by swapping movement direction and movement amount. Specifically, in this experiment, the discrete display scrolled horizontally (HDS) and the continuous display scrolled vertically (VCS). If scroll direction influences editing performance, then the results should be the opposite from those obtained in Experiment 1: more corrections in the continuous scrolling condition than in the discrete scrolling condition. However, if the amount of movement dominates editing performance, then the results should be the same those obtained in Experiment 1: more corrections in the discrete scrolling condition than in the continuous scrolling condition.

In addition, to add more update feedback to the serial presentation display, we modified the SP ticker to gradually fade headlines in and out. This effectively eliminates both motion and update feedback. If motion were more detrimental to performance than the lack of feedback, we would expect performance to be better for the fading display than the other tickers.

\section{Method}

As in Experiment 1, participants first performed the editing task without the headline-reading task. In the dual task condition, participants performed both tasks. Performance on the editing task alone served as the baseline to compare to editing performance in the dual-task conditions. After simultaneously performing the text-editing and headlinereading tasks, headline memorability was measured by a headline recall test. Unlike in the first experiment, participants in the second experiment saw all three tickers, as well as the no ticker condition. Thus, one withinsubjects factor, ticker type, was manipulated across three levels: vertical continuous scrolling (VCS), fading serial presentation (FSP), and horizontal discrete scrolling (HDS).

\section{Participants}

Twenty-three participants were recruited from a temporary employment agency and were compensated for their participation.

\section{Design and Dual-task Construction}

Each participant was exposed to all three tickers as well as the no ticker condition. For this design, three different sets of headlines were constructed along with three recall tests. Additionally, four different documents were created for editing (one in each condition). With the new design came three new factors, display order, document, and headline, which were randomized across participants. Distraction was measured as the number of corrections made within the ten-minute time period, and memorability was measured by the percent correct answers on a headline recall test.

\section{Materials}

Because differences had not been observed in memorability for Experiment 1, we attempted to increase the sensitivity of the test by changing it to a short answer fill-in-the-blank test (cued recall test). The documents used for editing in Experiment 1 were of moderately high difficulty. This may have contributed to somewhat higher variation in editing performance than expected. For Experiment 2, four new documents were chosen from popular press articles concerning current software tools or Internet applications. These documents were far easier to read (see Table 1). The method of introducing errors to the documents was the same as that used in the first experiment.

The FSP updated headlines centered in the display window. Headlines faded in by increasing the brightness of the text according to an exponential function, and then faded out by decreasing brightness according to the same function. The location and size of the text editor and headline display windows were the same as Experiment 1.

\section{Procedure}

Participants were presented with all four conditions of the experiment-no display, HDS, VCS, and FSP-in random order. The order of the four documents and the three sets of headlines were both randomized before the experiment began. In each condition, participants edited a document for ten minutes. After all but the "no ticker" condition, the appropriate recall test was administered. Participants were instructed to do their best on the recall test and to guess if they did not know the answer.

\section{Results}

Participants were required to surpass a minimum criterion of editing competency to be included in the analysis. Even reading at a slow pace, a participant should be able to read about four sentences per minute. Because each sentence contained between zero and two errors and the errors were evenly spaced throughout each document, participants would be expected to find at least one error every four sentences. Thus, our minimum performance criterion was 1 error per minute (and 10 errors in ten minutes) in the no display condition. Five of the twenty-three participants failed to meet this minimum criterion and were not considered further.

A one-way repeated measures ANOVA was calculated across the four within-subjects conditions, showing a significant effect on number of corrections, $F(3,51)=$ $20.659, p<0.0001$. The average number of corrections dropped from 24.67 in the baseline condition to 15.00 in the ticker conditions. The percentage decrease in the number of corrections from the baseline to the dual-task conditions is shown in Figure 3. Paired samples $t$-tests showed significantly more corrections in the HDS condition than the VCS condition, $t(17)=267, p=0.016$, but neither 
of the other comparisons (HDS vs. FSP, and VCS vs. FSP) were significant.

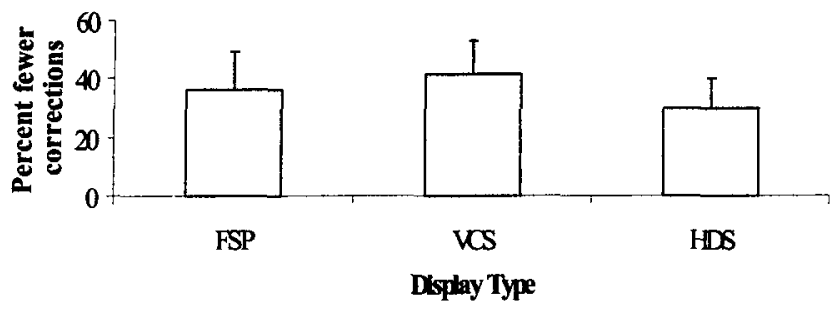

Figure 3: Percentage decrease in corrections between single- and dual-task conditions in Experiment 2.

Scores on the recall test measured memorability of the headlines. Once again, no difference was found among the displays, $F(2,51)=0.6$, NS. Mean scores were $58 \%$ for FSP, $56 \%$ VCS, and $49 \%$ for HDS.

\section{Discussion}

The results here support our original interpretation of the results of Experiment 1: Display movement and update feedback - rather than display direction-affect editing performance. As discussed, displays with less movement ought to be less distracting, enabling participants to remain primarily focused on the editing task. At the same time, tickers with less movement ought to provide more update feedback, thus helping participants efficiently schedule their glances at the ticker.

Although Experiment 2 ruled out motion direction, distraction and feedback were not clearly distinguished. The FSP condition in this experiment was intended to provide somewhat less feedback than the original SP condition of the first experiment. Yet, because the headlines on the FSP display does not provide update feedback, it is possible that FSP is more distracting than SP. Because of their different designs and different details, it would be inappropriate to compare conditions across these experiments directly. However, the ordering of the conditions by editing performance obtained in Experiment 1 (Figure 2) can be informally compared to the ordering obtained in Experiment 2 (Figure 3), revealing the same relative ordering. Although SP clearly differed from the discrete and continuous tickers in Experiment 1, FSP did not differ significantly from either the discrete or continuous conditions in Experiment 2. Thus, the fading condition served to muddle rather than to clarify the relationship between distraction and feedback.

\section{EXPERIMENT 3}

The third experiment tested whether distraction or feedback dominates performance by introducing conditions that provide feedback independent of motion. In particular, conditions in which simple visual and auditory highlighting provided update feedback were created. Whereas continuous scrolling displays move without providing explicit update feedback, and discrete scrolling displays move and provide explicit update feedback at the same time, visual and auditory highlighting provide explicit update feedback to a continuous scrolling display independent of display motion. In the visual highlighting condition, the background of the ticker display flashed briefly when a new headline was fully visible. In the auditory highlighting condition, a simple beep alerted participants when a new headline was fully visible. Because these methods for highlighting or providing update feedback do not involve motion, it should be possible to determine whether feedback or distraction dominates performance.

\section{Method}

As in the first experiment, a between-subjects design was used. In this case, participants first edited text without reading headlines. In the dual-task condition, participants saw one of four possible tickers: discrete scrolling (DS), continuous scrolling (CS), continuous scrolling with visual feedback (VF), and continuous scrolling with auditory feedback (AF). As in the two previous experiments, the effect of the headline-reading task was measured as the decrease in performance in the dual-task compared to the single-task conditions. As in Experiment 2, memorability of the headlines was measured by a recall test administered after the dual-task condition.

\section{Participants}

Forty undergraduates at the University of California, Santa Cruz participated for psychology course credit.

\section{Materials and Procedure}

The materials and procedure were the same as in the previous experiments with the following exceptions. Only articles 1 and 2 from Experiment 2 and the two sets of headlines from Experiment 1 were used. The procedure was the same as that of Experiment 1.

In all conditions, headlines scrolled from right to left. In the CS condition, text scrolled continuously, as in the HCS condition of Experiment 1. In the DS condition, headlines scrolled discretely, as in the HDS condition of Experiment 2. The VF condition was similar to the CS condition in that the text scrolled continuously left to right, but differed in that the background black turned yellow for $500 \mathrm{~ms}$ when a headline was fully displayed. Visually, the AF condition was identical to the CS condition, but when a headline was fully displayed, a low beep sounded.

\section{Results}

Four outliers-whose editing performance exceeded two standard deviations from the mean-were removed from further analysis, leaving nine participants in each of the four groups.

To test for differences among the four information displays, a one-way analysis of variance was calculated with percent decrease in number of edits as the dependent measure. A main effect was found for editing performance, $F(3,32)=$ 
$10.30, p<0.0001$. Figure 4 shows the mean percent decrease in number of corrections made between single-task and dual-task conditions. Planned comparisons using independent samples $t$-tests indicated: (a) discrete scrolling (DS) differed from continuous scrolling (CS), $t(16)=2.44$, $p=0.027$; (b) discrete scrolling (DS) differed from visual feedback (VF), $t(16)=4.01, p=0.001$; (c) continuous scrolling (CS) did not differ from visual feedback (VF), $t(16)=0.30, \mathrm{NS} ;$ (d) auditory feedback (AF) differed from continuous scrolling (CS), $t(16)=2.37, p=0.03$; and (e) visual feedback (VF) differed from auditory feedback (AF), $t(16)=2.86, p=0.01$. Overall, DS decreased performance least (26\%), VF (42\%) and CS (43\%) had a greater impact, and $\mathrm{AF}(55 \%)$ led to the largest decrease in performance.

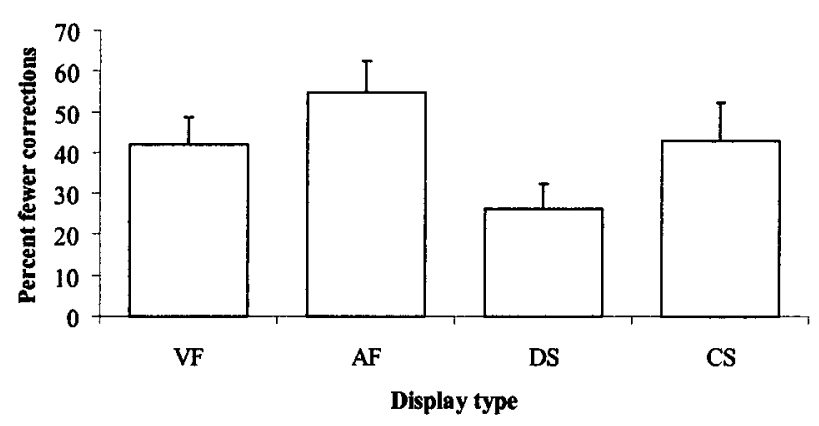

Figure 4 Percentage decrease in corrections between single- and dual-task conditions in Experiment 3.

As in the first two experiments, no difference was found among the displays in memorability, $F(3,32)=1.16$, NS. Mean scores were $43 \%$ for DS, $36 \%$ for CS, $42 \%$ for AF, $53 \%$ for VF.

\section{Discussion}

The effect of display type shows that display motion rather than update feedback dominates performance. Because the number of corrections decreased less (relative to baseline) for the discrete scrolling display than for continuous scrolling displays in which update feedback and motion were separate, we can conclude that continuous motion is more distracting than discrete motion. Moreover, because the auditory and visual feedback conditions led to worse editing performance than the discrete scrolling condition, we can conclude that update feedback is less important than display motion or distraction. Finally, because the headlines presented by all displays were equally memorable (as determined by the headline recall test), we can tentatively conclude that effective information delivery does not depend on distraction or feedback.

\section{GENERAL DISCUSSION}

This investigation was aimed at determining the properties of peripheral information displays that minimize distraction, maximize memorability, and cue effective multi-task scheduling strategies. Experiment 1 showed that a vertical, discrete scrolling headline display was as memorable as a horizontal, continuous scrolling display, but that the discrete display had less negative impact on a concurrent text-editing task. Experiment 2 showed that a horizontal, discrete scrolling display was as memorable as a vertical, continuous scrolling display, but that the discrete display had less impact on concurrent text editing. Experiment 3 showed that a discrete display was as memorable as a continuous display and less intrusive than a continuous display, regardless of external visual or auditory signals that new information is displayed. Taken together, these results demonstrate that motion of a scrolling display dominates performance on concurrent tasks. That is, constant motion of continuous scrolling displays distracts users from effectively performing other jobs at the same time. The start and stop motion of discrete scrolling displays distracts users far less under dual-task demands. What is more, the start and stop motion of the discrete display provides effective update feedback, enabling users to schedule their glances at the display.

Because continuous motion distracts computer users regardless of update feedback, it is likely that motion in the periphery captures users' attention too easily, leading to constant and costly task switching. Because discrete motion is not continual and the start-and-stop motion naturally provides update feedback, it does not constantly draw attention from other concurrent tasks and at the same time facilitates scheduling glances at the display. It seems as though discrete displays effectively balance motion and feedback in displaying peripheral information.

Although the serial presentation (SP) of Experiment 1 and the fading serial presentation (FSP) of Experiment 2 provide update feedback similar to that of discrete scrolling displays without adding motion, they led to worse editing performance than the discrete scrolling displays. One possible reason is that updates of the SP display were too brief to be noticed. Alternatively, the discrete updates of SP may have been too disruptive, creating higher taskswitching costs. Updates of the FSP display, by contrast, might have been too subtle, fading in so gradually that they did not provide appropriate update feedback. In any event, our data cannot decide among these alternatives.

\section{CONCLUSION}

As user interfaces attempt to present more and more information, computer screens often become cluttered and distracting, effectively eliminating a user's ability to accomplish a single task. As stated, the key to peripheral informing is to maximize the information presented while minimizing the presentation's impact on ongoing activities. In this paper, we have considered the peripheral display of information by investigating mental workload tradeoffs among several scrolling ticker displays. Our finding that distraction dominates performance in this context marks only the first step toward a full understanding peripheral information displays. Additional studies are needed to understand, for instance, the nature of interruptions and the details of how users actually shift attention to peripheral 
information. Nevertheless, based on our results, we can suggest some guidelines for designing peripheral displays.

1. Motion or animation should be kept at a minimum.

2. Motion or animation (when used) should not be continuous.

3. Discrete animation seems ideal for update feedback.

4. Visual feedback is better than auditory (for visual information).

5. Scrolling direction does not seem to matter.

Overall, we recommend the discrete scrolling display as the best way to convey memorable yet peripheral information.

\section{ACKNOWLEDGMENTS}

Thanks to Teenie Matlock, Michael Wenger, and Shumin Zhai for helpful comments on a draft of this paper.

\section{REFERENCES}

1. Adorni, G., \& Poggi, A. (1996). Route guidance as a just-in-time multiagent task. Applied Artificial Intelligence, 10, 95-120.

2. Campbell, C. S. \& Maglio, P. P. (1999). Facilitating navigation in information spaces: Road signs on the World Wide Web. International Journal of HumanComputer Studies, 50, 309--327.

3. Cypher, A. (1986). The structure of users' activities. In D. A. Norman \& S. W. Draper (Eds.), User centered system design (pp. 243-263). Hillsdale, NJ: LEA.

4. Damos, D. L. \& Wickens, C. D. (1980). The identification and transfer of timesharing skills. Acta Psychologica, 46, 15-39.

5. Duchnicky, R. L. \& Kolers, P. A. (1983). Readability of text scrolled on visual display terminals as a function of window size. Human Factors, 25, 683-692.

6. Flesch, R. (1948). A new readability yardstick. Journal of Applied Psychology, 32, 221-233.

7. Gopher, D. \& North, R. A. (1977). Manipulating the conditions of training in time-sharing performance. Human Factors, 19, 583-594.

8. Granaas, M. M., McKay, T. D., Laham, R. D., Hurt, L. D. \& Juola, J. F. (1984). Reading moving text on a CRT screen. Human Factors, 26, 97-104.

9. Ishii, H. \& Ullmer, B. (1997). Tangible bits: Towards seamless interfaces between people, bits, and atoms. In Proceedings of CHI '97 (pp. 234-241), New York: ACM Press.

10. Juola, J. F., Ward, N. J., \& McNamara, T. (1982). Visual search and reading of rapid serial presentations of letter strings, words, and text. Journal of Experimental Psychology: General, 111, 208-227.
11.Kanarick, A. F. \& Petersen, R. C. (1969). Effects of value on the monitoring of multi-channel displays. Human Factors, 11, 313-320.

12.Kang, T. J. \& Muter, P. (1989). Reading dynamically displayed text. Behaviour \& Information Technology, $8,33-42$

13. Kramer, A. F., Larish, J. F., \& Strayer, D. L. (1995). Training for attentional control in dual-task settings: A comparison of young and old adults. Journal of Experimental Psychology: Applied, 1, 50-76

14.Lee, E. S., Okada, R., \& Jeon, I. G. (1997). Agent-based support for personalized information with Web search engines. In Proceedings of HCI International 97 (pp. 783-786), Elsevier.

15.Lieberman, H. (1997). Autonomous interface agents. In Proceedings of CHI 97 (pp. 67-74), New York: ACM Press.

16. Maglio, P. P. (1995). The computational basis of interactive skill. Unpublished doctoral dissertation, University of California, San Diego.

17. Maglio, P. P. \& Barrett, R. (1997). How to build modeling agents to support web searchers. In Proceedings of User Modeling 97 (pp. 5-16), New York: Springer Wein.

18. Moray, N. (1986). Monitoring behavior and supervisory control. In K. R. Boff, L. Kaufman, \& J.P. Thomas (Eds.), Handbook of Perception and Human Performance: v. II, New York: Wiley.

19. Norman, D. A. (1986). Cognitive engineering. In D. A. Norman \& S. W. Draper (Eds.), User centered system design, Hillsdale NJ: LEA.

20. Owen, D. (1986). Answers first, then questions. In D. A. Norman \& S. W. Draper (Eds.), User centered system design (pp. 361-375). Hillsdale, NJ: LEA.

21.Potter, M. C., Kroll, J. F. \& Harris, C. (1980). Comprehension and memory in rapid sequential reading. In R. Nickerson (Ed.), Attention and Performance VIII. Hillsdale, NJ: Erlbaum.

22. Sekey, A. \& Tietz, J. (1982). Text display by 'saccadic scrolling'. Visible Language, 16, 62-76.

23. Wickens, C. D. (1992). Engineering psychology and human performance (2nd Edition). New York, NY: Harper Collins.

24. Wickens, C. D. \& Seidler, K. S. (1997). Information access in a dual-task context: Testing a model of optimal strategy selection. Journal of Experimental Psychology: Applied, 3, 196-215. 